1 Synthesis and Optoelectronic Properties of a Solution-processed Red-emitting

\title{
Tetra(arylvinyl)anthracene Cruciform
}

Bing Hua, ${ }^{\mathrm{a}}$ Yangyang Gao, ${ }^{\mathrm{a}}$ ShianYing, ${ }^{\mathrm{a}}$ Wei Liu, ${ }^{\mathrm{a}}$ Shanfeng Xue, ${ }^{\mathrm{ab}}$ Wenjun Yang $*^{\mathrm{a}}$ .

${ }^{a}$ Key Laboratory of Rubber-Plastics of Ministry of Education/Shandong Province (QUST), School of Polymer Science \& Engineering, Qingdao University of Science \& Technology, 53-Zhengzhou Road, Qingdao 266042, China

${ }^{b}$ State Key Laboratory of Luminescent Materials and Devices, Institute of Polymer Optoelectronic Materials and Devices, South China University of Technology, Guangzhou 510640, P. R. China.

3

\section{Abstract:}

In this paper, we report the synthesis and optoelectronic properties of a new anthracene-centered cruciform, 2,6-bis(9,9-di(2-ethylhexyl)fluorene-2-yl-vinyl-2)9,10-bis(9-(2-ethylhexyl)carbazole-3-yl-vinyl-2)anthracene $\quad$ (FCA). FCA was prepared by the Heck coupling 2,6-bis(diethoxylphosphorylmethyl)-9,10dibromoanthracene and 9-(2-ethylhexyl)-3-vinylcabazole, followed by the Wittig-Horner reaction with 9,9-di(2-ethylhexyl)-2-formylfluorene. The existence of strongly twisted 9,10-branch made FCA exhibit moderate aggregation-enhanced emission and piezofluorochromism, and the introduction of numerous branched alkyl chains render FCA with good solubility and film-forming ability. FCA could be used 
as solution-processed light-emitting layer to afford red emission with peak wavelength of $612 \mathrm{~nm}$ and maximal luminous efficacy of $1.3 \mathrm{~cd} / \mathrm{A}$.

Keywords: Center-crossed conjugated molecule; Anthracene-based cruciform; Piezofluorochromism; Solution-processed oligomer; Aggregation-enhanced emission; Solid-state red emitting material.

\subsection{Introduction:}

Conjugated organic molecules have attracted much attention due to their potential applications in organic optical and optoelectronic fields, and much effort has devoted to the design and synthesis of new luminogens with unique structures and optoelectronic properties. While the linear $\pi$-conjugated organic molecules have been the mainstay of optical and optoelectronic materials, recently, higher orders, especially two dimensional arylene-centered oligomers, have started to draw serious research interest, and some benzene-, thiophene-, anthracene-, pyrazine-, benzobisoxazole-, and benzobisthiophene-centered cruciforms have been designed, synthesized and their optical and optoelectronic properties investigated.[1-10] These new configuration materials with unique geometric and electronic structures have greatly widened the scope of molecular candidates applicable in chemosensors, molecular switches, nonlinear optical materials, field effect transistors, and photovoltaic and electroluminescence devices.[11-15]

We have been interested in 2,6,9,10-tetra(arylvinyl)anthracene derivatives due to their unique $\pi$-centered structure and distorted conformation. Compared to the 
extensively investigated benzene-centered and other aryl-centered analogues, 2,6,9,10-tetra(arylvinyl)anthracene cruciforms have an asymmetric $\pi$-center and a strongly twisted 9,10-branch, which could render them with unique aggregation behaviors and optical properties.[16-18] For example, donor- and/or acceptor-capped cruciforms show large and enhanced two-photon absorption cross sections, and pyrindine- and/or dibutylaniline-capped cruciforms exhibit the selectively fluorescence sensing on metal ions and piezofluorochromism based on 9,10-bis(arylvinyl)anthracene branch,[19-22] etc. Since center-crossed cruciforms have multiple conjugation pathways for intramolecular charge transfer, donor- and/or acceptor-substituted anthracene-centered cruciforms usually exhibit more bathochromic shift emission than their linear building blocks 9,10- and 2,6-bis(arylvinyl)anthracenes.[23-25] Moreover, the existence of twisted branch 9,10-bis(arylvinyl)anthracene could alleviate from intermolecular tight stacking and strong interactions, which should improve the solid-state fluorescence efficiency and could be promising light-emitting materials with unique structure.[26-27] However, such tetra(arylvinyl)anthracene cruciforms usually have large and rigid conjugation skeleton, which is not beneficial to solubility and film fabrication by vacuum deposition. In the current work, we have introduced several branched alkyl chains into the molecular peripheries to improve the solubility and the film-forming capacity. We now report the synthesis and optoelectronic properties of a new and solution-processed 2,6,9,10-tetra(arylvinyl)anthracene cruciform, 2,6-bis(9,9-di(2ethylhexyl)fluorene-2-yl-vinyl-2)-9,10-bis(9-(2-ethylhexyl)carbazole-3-yl-vinyl-2)- 
anthracene (FCA).

\subsection{Experimental Section}

\subsubsection{Materials}

Acetonitrile (MeCN) and tetrahydrofuran (THF) (over metallic sodium) and triethylamine (over $\mathrm{CaH}_{2}$ ) were distilled before use. Carbazole, 2-bromofluorene and other solvents and reagents (analytical grade) were commercial available and used as received, unless otherwise claimed. 2,6-Bis(diethylphosphorylmethyl)- 9,10-dibromoanthracene was from the previous work.[28]

\subsubsection{Preparation of aqueous dispersion and ground sample.}

The stock solution of FCA in THF with a concentration of $1.0 \times 10^{-4} \mathrm{M}$ was prepared and reserved. THF/water mixtures with different water fractions (aqueous dispersion) were prepared by slowly adding distilled water into the THF solution of FCA under ultrasound at room temperature, and the final concentration was kept at $1.0 \times 10^{-5} \mathrm{M}$. Ground sample was prepared by grinding FCA powder on a glass plate with a metal spatula at room temperature.

\subsubsection{Measurements}

${ }^{1} \mathrm{H}$ and ${ }^{13} \mathrm{C}$ NMR spectra were recorded on a Bruker-AC500 (500 MHz) spectrometer with $\mathrm{CDCl}_{3}$ as solvent and tetramethylsilane (TMS) as the internal standard. Elemental analysis was performed on a Perkin-Elmer 2400. UV-vis absorption spectra were recorded on a Hitachi U-4100 spectrophotometer. Emission spectra were measured with a Hitachi F-4600 spectrophotometer. The peak 
wavelength of the lowest energy absorption band was used as the excitation wavelength for the PL measurement. The fluorescence quantum yield $(\Phi)$ was determined at room temperature by the dilution method using rhodamine $\mathrm{B}$ in methanol as the reference. [29] Cyclic voltammetry (CV) was performed with a BAS 100W Bioanalytical Systems, using a glass carbon disk (diameter $=3 \mathrm{~mm})$ as the working electrode, a platinum wire as the auxiliary electrode with a porous ceramic wick, and $\mathrm{Ag} / \mathrm{Ag}^{+}$as the reference electrode standardized by the redox couple ferrocenium/ferrocene. All solutions were purged with a nitrogen stream for $10 \mathrm{~min}$ before measurement. The procedure was performed at room temperature, and a nitrogen atmosphere was maintained over the solution during measurements. [30-32] Differential scanning calorimetry (DSC) and thermogravimetric (TG) analysis were performed under nitrogen atmosphere on Netzsch DSC 204F1 and Netzsch 209F1 analyzers at heating rates of $10^{\circ} \mathrm{C} / \mathrm{min}$ and $20^{\circ} \mathrm{C} / \mathrm{min}$, respectively.

\subsubsection{Device Fabrication}

Indium-tin oxide (ITO) coated glass with a sheet resistance of $15-20 \Omega / \mathrm{cm}^{2}$ was used as the cathode whose substrate was pre-patterned by photolithography to give an effective device size of $6.25 \mathrm{~mm}^{2}$. Then, it was cleaned in an ultrasonic bath with acetone, detergent, deionized water, and isopropanol as the clean agent. After being dried in an oven, it is treated with oxygen plasma for $4 \mathrm{~min}$, and then $40 \mathrm{~nm}$ of PEDOT:PSS layer was spin-coated and baked overnight in a vacuum oven at $80{ }^{\circ} \mathrm{C}$. On the top of above active layer, a $60 \mathrm{~nm}$ of FCA bulk emitting layer was spin-casted 
111 from its chloroform solution $(12 \mathrm{mg} / \mathrm{mL})$. The FCA solution in chloroform has been

112 filtered through a $0.45 \mu \mathrm{m}$ PTFE filter before spin coating. Then 1,3,5-tri(phenyl-2-

113 benzimidazol-yl)benzene (TPBi) as electron-transporting layer (50 nm) were vacuum

114 deposited. Finally, $0.5 \mathrm{~nm}$ of LiF film and $100 \mathrm{~nm}$ of $\mathrm{Al}$ film was vacuum deposited to

115 form the anode. The base pressure for vacuum deposition was at of $3 \times 10^{-4} \mathrm{~Pa}$. The

116 thickness of each deposition layer was monitored using a quartz crystal thickness/ratio

117 monitor (STM-100/MF, Sycon). Electroluminescence (EL) spectra were measured by

118 a PR650 fluorescence spectrophotometer. Luminance-voltage and current density-

119 voltage characteristics were recorded simultaneously by combining the spectrometer

120 with a Keithley model 2400 programmable voltage-current source. All measurements

121 were carried out at room temperature under ambient conditions.

$122 \quad$ 1.2.5 Synthesis

123

124

125

126

127

9,10-Bis(9-(2-ethylhexyl)carbazole-3-yl-vinyl-2)-2,6-bis(diethylphosphorylmethyl)-

anthracene

A dried pressure tube was added 2,6-bis(diethylphosphorylmethyl)-9,10-dibromoanthracene (0.21 g, $0.33 \mathrm{mmol})$, 9-(2-ethylhexyl)-3-vinyl-carbazole (0.36 g, 1.18 $\mathrm{mmol}), \mathrm{Pd}(\mathrm{OAc})_{2}(7.4 \mathrm{mg}, 0.033 \mathrm{mmol})$, tri $(o$-toyl $)$ phosphine $(68 \mathrm{mg}, 0.23 \mathrm{mmol})$, $\mathrm{N}, \mathrm{N}$-dimethylformamide (DMF) $(1.5 \mathrm{~mL})$, and triethylamine $(1.5 \mathrm{~mL})$, and then purged by nitrogen and sealed with a Teflon screw top. The mixture was refluxed for $24 \mathrm{~h}$ and then extracted with dichloromethane. The solvent was removed and the crude product was separated by column chromatography on silica gel using petroleum ether/ethyl acetate (1/1) as the eluent. Yield: $0.21 \mathrm{~g}(58 \%) .{ }^{1} \mathrm{H}$ NMR (500 MHz, 
133

$\left.\mathrm{CDCl}_{3}, \mathrm{ppm}\right): \delta 8.48(\mathrm{~d}, 2 \mathrm{H}), 8.38(\mathrm{~s}, 2 \mathrm{H}), 8.34(\mathrm{~s}, 2 \mathrm{H}), 8.18(\mathrm{~d}, 2 \mathrm{H}), 7.93(\mathrm{~d}, 2 \mathrm{H})$, $7.86(\mathrm{~d}, 2 \mathrm{H}), 7.49(\mathrm{~m}, 8 \mathrm{H}), 7.28(\mathrm{~d}, 2 \mathrm{H}), 7.12(\mathrm{~d}, 2 \mathrm{H}), 4.24(\mathrm{~m}, 4 \mathrm{H}), 4.03(\mathrm{~m}, 8 \mathrm{H})$, $3.36(\mathrm{~d}, 4 \mathrm{H}), 2.13(\mathrm{~m}, 2 \mathrm{H}), 1.22(\mathrm{~m}, 16 \mathrm{H}), 0.96(\mathrm{t}, 12 \mathrm{H}), 0.90(\mathrm{t}, 12 \mathrm{H})$.

2,6-Bis(9,9-di(2-ethylhexyl)fluorene-2-yl-vinyl-2)-9,10-bis(9-(2-ethylhexyl)carbazole3-yl-vinyl-2)anthracene (FCA)

Potassium tert-butoxide $(0.16 \mathrm{~g}, 1.43 \mathrm{mmol})$ was added to a dried one-neck flask containing the solution of 9,10-bis(9-(2-ethylhexyl)carbazole-3-yl-vinyl-2)-2,6-bis(diethylphosphorylmethyl)anthracene $(0.17 \mathrm{~g}, 0.16 \mathrm{mmol})$ and 9,9-di(2-ethylhexyl)2-formylfluorene $(0.19 \mathrm{~g}, 0.45 \mathrm{mmol})$ in anhydrous THF $(10 \mathrm{~mL})$ under nitrogen. The mixture was stirred over night at room temperature and then $100 \mathrm{~mL}$ of methanol was added. The precipitate was collected and purified by a column chromatography on silica gel using petroleum ether/dichloromethane (2/1) as the eluent. Yield: $0.21 \mathrm{~g}$ (82\%). ${ }^{1} \mathrm{H}$ NMR (500 MHz, $\left.\mathrm{CDCl}_{3}, \mathrm{ppm}\right): \delta 8.52(\mathrm{~d}, 2 \mathrm{H}), 8.48(\mathrm{~s}, 2 \mathrm{H}), 8.42(\mathrm{~s}, 2 \mathrm{H})$, $8.22(\mathrm{~d}, 2 \mathrm{H}), 8.01(\mathrm{~d}, 2 \mathrm{H}), 7.94(\mathrm{~d}, 2 \mathrm{H}), 7.86(\mathrm{~d}, 2 \mathrm{H}), 7.64(\mathrm{t}, 4 \mathrm{H}), 7.58(\mathrm{t}, 2 \mathrm{H}), 7.50$ (m, 8H), $7.30(\mathrm{~m}, 10 \mathrm{H}), 7.23(\mathrm{~m}, 4 \mathrm{H}), 4.27(\mathrm{~d}, 4 \mathrm{H}), 2.16(\mathrm{t}, 2 \mathrm{H}), 1.98(\mathrm{~m}, 8 \mathrm{H}), 1.05$ $(\mathrm{m}, 4 \mathrm{H}), 0.80(\mathrm{~m}, 12 \mathrm{H}), 0.75(\mathrm{~m}, 12 \mathrm{H}), 0.70(\mathrm{t}, 6 \mathrm{H}), 0.65(\mathrm{~m}, 24 \mathrm{H}), 0.56(\mathrm{t}, 6 \mathrm{H}), 0.48$ (t, 24H). ${ }^{13} \mathrm{C}$ NMR $\left(125 \mathrm{MHz}, \mathrm{CDCl}_{3}, \mathrm{ppm}\right): \delta 150.98,150.67,141.42,141.11$, $141.00,140.91,138.29,135.82,135.77,135.71,134.20,133.03,130.28,129.64$, $129.58,128.57,128.12,127.29,126.69,126.36,126.10,125.92,124.47,124.01$, $123.29,122.85,122.19,121.71,120.51,119.71,119.55,119.12,118.78,109.31$, $109.22,54.75,47.56,44.61,39.49,34.51,33.56,31.93,29.71,28.88,27.00,26.85$, $24.42,23.11,22.68,14.80,14.00,10.34$. 


\section{9-(2-ethylhexyl)-3-formylcarbazole}

To a cooled flask in an ice bath was added phosphoryl chloride $(0.6 \mathrm{~mL}, 5.9 \mathrm{mmol})$ and anhydrous DMF (1.4 mL, $17.7 \mathrm{mmol})$ under nitrogen. To this mixture was added 9-(2-ethylhexyl)carbazole $(1.5 \mathrm{~g}, 5.4 \mathrm{mmol}$, in $20 \mathrm{~mL}$ sym-dichloroethane). After stirring for $1 \mathrm{~h}$, the reaction temperature was raised to $90{ }^{\circ} \mathrm{C}$ and left for $8 \mathrm{~h}$. The mixture was cooled and poured into ice water and extracted with dichloromethane.

The solvent was evaporated and the crude product was purified by column chromatography on silica gel using ethyl acetate/hexane (1/10) as the eluent. Yield: $1.3 \mathrm{~g}(78.3 \%) .{ }^{1} \mathrm{H}$ NMR (500 MHz, $\left.\mathrm{CDCl}_{3}, \mathrm{ppm}\right): \delta 10.08(\mathrm{~s}, 1 \mathrm{H}), 8.60(\mathrm{~s}, 1 \mathrm{H}), 8.15$ $(\mathrm{d}, 1 \mathrm{H}), 8.01(\mathrm{~d}, 1 \mathrm{H}), 7.54(\mathrm{t}, 1 \mathrm{H}), 7.47(\mathrm{t}, 2 \mathrm{H}), 7.33(\mathrm{t}, 1 \mathrm{H}), 4.35(\mathrm{~d}, 2 \mathrm{H}), 1.95(\mathrm{~m}$, $1 \mathrm{H}), 1.50(\mathrm{~m}, 4 \mathrm{H}), 1.23(\mathrm{~m}, 4 \mathrm{H}), 0.95(\mathrm{t}, 6 \mathrm{H})$.

9,9-Di(2-ethylhexyl)-2-formylfluorene

To a solution of 2-bromo-9,9-di(2-ethylhexyl)-fluorene $(3.5 \mathrm{~g}, 6.03 \mathrm{mmol})$ in anhydrous THF $(15 \mathrm{~mL})$ was added dropwise $n$-BuLi $(2.5 \mathrm{~mL}, 7.24 \mathrm{mmol})$ at $-78^{\circ} \mathrm{C}$. After stirring for $1 \mathrm{~h}$, anhydrous DMF (2.87 g, $35.0 \mathrm{mmol})$ was added and stirred for an additional $12 \mathrm{~h}$ at $0{ }^{\circ} \mathrm{C}$. The resulting mixture was added to water and extracted with dichloromethane. The organic layer was separated and washed with diluted $\mathrm{HCl}$ and then saturated aqueous $\mathrm{NaHCO}_{3}$. The solvent was removed by evaporation and the residue was purified by column chromatography using dichloromethane/petroleum ether (1/10) as the eluent to give a colorless oil. Yield: $1.43 \mathrm{~g}(45 \%) .{ }^{1} \mathrm{H}$ NMR (500 $\left.\mathrm{MHz}, \mathrm{CDCl}_{3}, \mathrm{ppm}\right): \delta 10.05(\mathrm{~s}, 1 \mathrm{H}), 7.85(\mathrm{~d}, 1 \mathrm{H}), 7.80(\mathrm{t}, 1 \mathrm{H}), 7.77(\mathrm{t}, 1 \mathrm{H}), 7.70(\mathrm{~d}$, 1H), $7.67(\mathrm{~s}, 1 \mathrm{H}), 7.61(\mathrm{~d}, 1 \mathrm{H}), 7.55(\mathrm{~d}, 1 \mathrm{H}), 1.98(\mathrm{~d}, 4 \mathrm{H}), 1.50(\mathrm{~m}, 2 \mathrm{H}), 1.23(\mathrm{~m}$, 


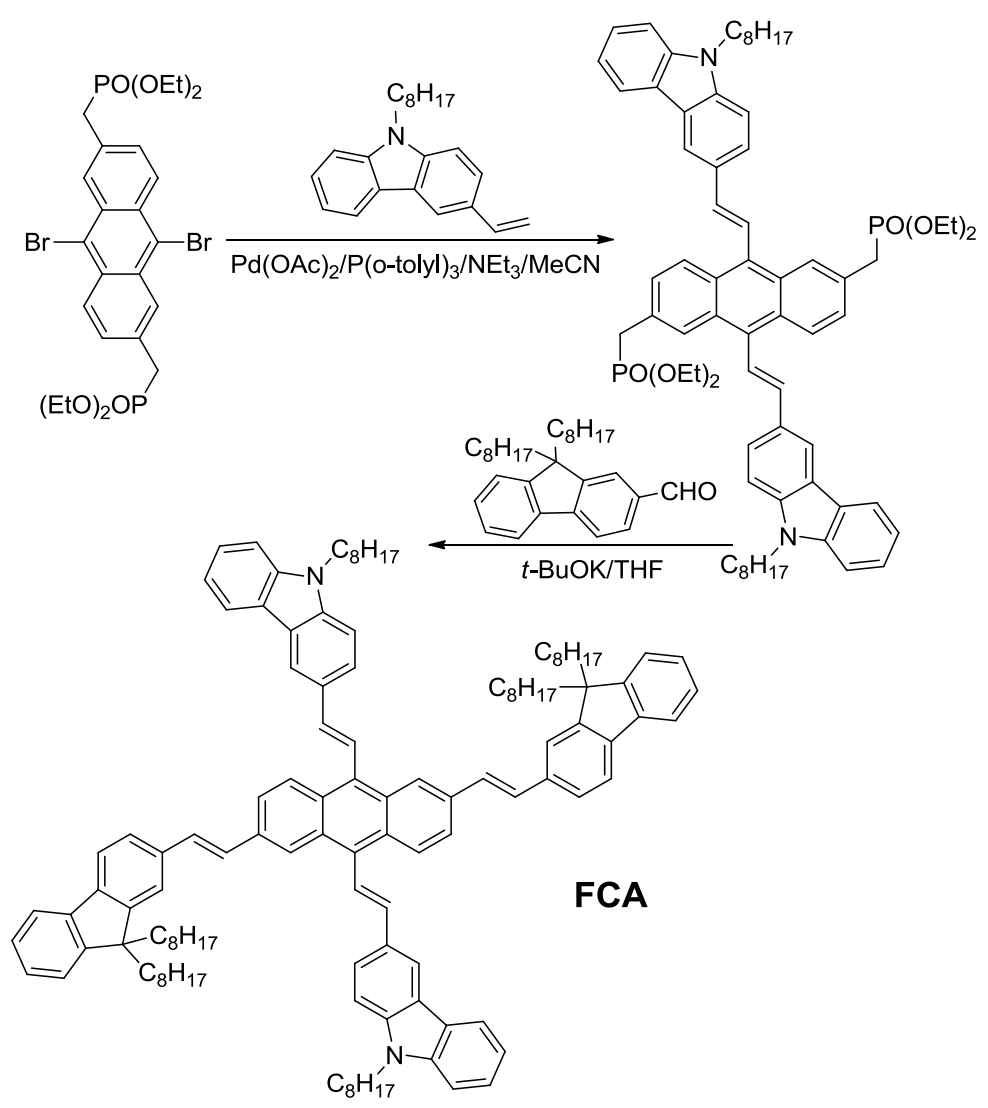

179 Scheme 1. The structure and synthetic route of anthracene-centered cruciform (FCA) studied in this paper.

\subsection{Results and discussion}

\subsubsection{Synthesis and characterization}

Carbazole- and/or fluorene-capped 2,6,9,10-tetravinylanthracene derivatives have the large and rigid conjugation skeleton, which leads to the poor solubility in common organic solvents. Here we have introduced numerous branched alkyl chains (2-ethylhexyl) to the skeleton periphery in order to obtain highly soluble anthracene-based cruciform. Indeed, FCA is soluble in many common organic solvents, such as 
resulting solutions with $10 \mathrm{mg} / \mathrm{mL}$ could smoothly pass through $0.45 \mu \mathrm{m}$ PTFE filter. We choose the Heck coupling as the first step reaction, which produces the highly soluble and decreased polar intermediate containing 2,6-bis(diethylphosphinemethyl) moieties. After Wittig-Horner reaction, this polar intermediate is changed into weakly polar FCA. The differential polarities among them make the separation easy. The structure and composition of target cruciform FCA was unambiguously confirmed by NMR and elemental analysis.

\subsubsection{Emission spectra of solution and aqueous dispersion}

Fig. 1 shows the absorption and emission spectra of FCA in THF solution. There are two absorption bands whose peak wavelengths are at 385 and $459 \mathrm{~nm}$, respectively. The higher energy band is ascribed to arylvinyl moieties, and the lower one is from the intramolecular charge transfer (ICT) transition of whole $\pi$ system. FCA is a strong absorption chromophore with the molar extinction coefficient up to $8.97 \times 10^{4} \mathrm{M}^{-1} \mathrm{~cm}^{-1}\left(\varepsilon_{\max }\right.$ at $\left.459 \mathrm{~nm}\right)$. It is known that 9,10-bis(arylvinyl)anthracenes are weakly fluorescent and 2,6-bis(arylvinyl)anthracenes emit intensely in solutions. FCA shows the moderate intensity of emission with the peak wavelength of $566 \mathrm{~nm}$ and the fluorescence quantum yield of $12.7 \%$, indicating that the intramolecular torsion motion of 9,10-branch still plays a role of deceasing fluorescence emission. This could be further evidenced by its broad emission band implying the existence of large amplitude relaxation in the excited state.

Fig. 1 The absorption and emission spectra of FCA solution in THF at $1.0 \times 10^{-5} \mathrm{M}$. 
213 Fig. 2 shows the emission spectra of FCA in THF/water mixtures. When a large

214 amount of water is added to the THF solution of FCA, the emission intensity of

215 aqueous dispersion is gradually enhanced and reaches about three times higher than

216 that of pure THF solution. Since water is a non-solvent for FCA, the dye molecules

217 must have aggregated in THF/water mixtures with high water contents. Therefore,

218 FCA is still an aggregation-enhanced emission molecule although the limited

219 enhancement amplitude.

221 Fig. 2 The emission spectra of FCA in THF/water mixtures at $1.0 \times 10^{-5} \mathrm{M}$.

\subsubsection{Solid-state photophysical and thermal properties}

224 FCA is an aggregation-enhanced emission dye in THF/water mixtures, which might result in strong solid-state fluorescence emission. Fig. 3 shows the absorption and emission spectra of FCA under pristine (as-prepared) and ground solid-states. In the pristine solid state, FCA has an ICT absorption band centered at $458 \mathrm{~nm}$ and is an orange emitting material with peak wavelength of $578 \mathrm{~nm}$ and fluorescence efficiency of $11.4 \%$. In the ground solid state, the ICT absorption peak and emission spectrum,

230 relative to the pristine solid, are red-shifted about $10 \mathrm{~nm}$ and $23 \mathrm{~nm}$, respectively.

231 Ground FCA solid emits red fluorescence with peak wavelength of $601 \mathrm{~nm}$ and 232 fluorescence efficiency of 6.5\%. Nevertheless, FCA is still fluorescent but not strong. 233 It is known that a distorted geometry in the monomeric form (solution) limits 
234 effective conjugation. In the solid state, however, its conjugation degree increases by 235 stacking-induced planarization, which results in the bathochromic shift spectra. 236 Moreover, the still partially distorted structure resulting from the internal steric 237 hindrance of 9,10-branch, even after stacking, disturbs the close packing, to partially 238 diminish the intermolecular quenching effects induced by planar 2,6-branch. In this 239 context, if 2,6-branches are also designed to be distorted structure, the cruciforms 240 with high fluorescence efficiencies could be expected.

Fig. 3 The absorption and emission spectra of FCA under pristine (as-prepared) and ground solid-states. exothermic transition centered at $187^{\circ} \mathrm{C}$ for ground solid. The two thermal transitions could be ascribed to the glass transition and the followed cold-crystallization of 
amorphized FCA solid induced by grinding. This indicates that pristine and ground FCA solids are in crystalline and amorphous states, respectively, and mechanical grinding could cause the changes of aggregate morphologies and spectral shifts, rendering FCA solid piezofluorochromism.

Fig. 4 The thermogravimetric (TGA) curve of FCA solid and the differential scanning calorimetric (DSC) curves of FCA solids under pristine (as-prepared) and ground solid-states (inset).

\subsubsection{Electrochemical and Electroluminescence properties}

Fig. 5 shows the cyclic voltammetry (CV) curves of FCA using $0.1 \mathrm{M}$ tetrabutylammonium hexafluorophosphate as supporting electrolyte in acetonitrile, with silver-button working electrodes, a silver-wire counter electrode, and a saturated calomel electrode (SCE) reference electrode. The SCE reference electrode was calibrated using the ferrocene/ferrocenium $\left(\mathrm{Fc} / \mathrm{Fc}^{+}\right)$redox couple as an external standard. As shown in Fig. 5, the reversible oxidative and reductive behaviors are observed and indicate that they could transport the hole and electron. The oxidative and reductive potentials are measured to be $\mathrm{E}_{\mathrm{ox}}=0.38 \mathrm{eV}$ and $\mathrm{E}_{\mathrm{red}}=-1.87 \mathrm{eV}$, respectively. Based on the onset oxidative and reductive potentials, the HOMO and LUMO energy levels are estimated to be -4.97 and $-2.84 \mathrm{eV}$, respectively, and the corresponding band gap is calculated as $2.13 \mathrm{eV}$. 
Fig. 5 The cyclic voltammetry (CV) curves of FCA in MeCN

While some tetra(arylvinyl)anthracene-based cruciforms have been investigated to show two-photon absorption, fluorescence sensing metal ions, piezofluorochromism, their application in electroluminescence has rarely been explored. In order to examine the EL performance, a device with the configuration of ITO/PEDOT:PSS (40 nm)/FCA $(60 \mathrm{~nm}) / \mathrm{TPBi}(40 \mathrm{~nm}) / \mathrm{LiF}(0.5 \mathrm{~nm}) / \mathrm{Al}(100 \mathrm{~nm})$ has been fabricated. Fig. 6 shows the current density-luminance-voltage characteristics. The device emits red light (at $612 \mathrm{~nm}$ with the Commission Internationale d'Eclairage Coordinates of ( 0.58 , 0.41), the inset in Fig. 7) with the maximal luminance of $1300 \mathrm{~cd} / \mathrm{cm}^{2}$ and the turn-on voltage of 4.2 V. The EL efficiency-voltage curve is plotted in Fig. 7. The luminous and power efficiencies are $1.31 \mathrm{~cd} / \mathrm{A}$ and $0.51 \mathrm{~lm} / \mathrm{W}$, respectively. Although this EL performance itself is not impressive, [34] it is the best one among the solution-processed red fluorescent small-molecular OLEDs based on anthracene-centered cruciform molecules. It should be noted that the fluorescence efficiency of FCA in amorphous state is only 6.5\%. This is probably the key reason for the low EL efficiency, and the better EL performances could be expected if cruciform's solid-state fluorescence efficiencies are improved. This could be achieved by introducing cyano groups to the vinyl bond of 2,6-branch to further distort the cruciform skeleton structure, which is underway in our laboratory.

Fig. 6 Current density-luminance-voltage curves of the device ITO/PEDOT:PSS (40 
nm)/ FCA $(60 \mathrm{~nm}) / \mathrm{TPBi}(40 \mathrm{~nm}) / \mathrm{LiF}(0.5 \mathrm{~nm}) / \mathrm{Al}(100 \mathrm{~nm})$. ITO/PEDOT:PSS (40 nm)/ FCA (60 nm)/TPBi $(40 \mathrm{~nm}) / \mathrm{LiF}(0.5 \mathrm{~nm}) / \mathrm{Al}(100 \mathrm{~nm})$. The inset is the electro- luminescent spectrum.

\subsection{Conclusions}

FCA soluble in common organic solvents, rendering FCA with good film-forming capacity. FCA exhibits moderate aggregation-enhanced emission and piezofluorochromic properties, which could be ascribed to strongly twisted 9,10-branch. A

313 solution-processed non-doped device affords red EL emission with the maximal

314 luminance of $1300 \mathrm{~cd} / \mathrm{cm}^{2}$ and the luminous efficiency of $1.31 \mathrm{~cd} / \mathrm{A}$, which is the best performance among solution-processed anthracene-based materials. We believe that better EL performances could be obtained if cruciform's solid-state fluorescence

317 efficiencies are improved by introducing cyano groups to the vinyl bond of 2,6-branch 318 to further distort the cruciform skeleton, which is underway in our laboratory.

\section{Acknowledgment}

321 We are grateful for the financial support from the NSF of China (51173092, 
51073083, and 51303091), the NSF of Qingdao City (13-1-4-207-jch) and the Specialized Research Fund for the Doctoral Program of Higher Education (no. 20133371912 0005). We also thank the State Key Laboratories of Luminescent Materials and Devices of South China University of Technology (SKLLMD201408). and the State Key Laboratory of Supramolecular Structure and Materials of Jilin University (SKLSSM2015023).

\section{References}

[1] Okamoto K, Zhang Jun, Housekeeper J, Marder S, Luscombe C. C-H arylation reaction: atom efficient and greener syntheses of $\pi$-conjugated small molecules and macromolecules for organic electronic materials. Macromolecules 2013;46:8059-78.

[2] Zerdan R, Cohn P, Puodziukynaite E, Baker M, Voisin M, Sarun C, et al. Synthesis, optical properties, and electronic structures of nucleobase-containing $\pi$-conjugated oligomers. J Org Chem 2015;80:1828-40.

[3] Zucchero A, Wilson J, Bunz U. Cruciforms as functional fluorophores: response to protons and selected metal ions. J Am Chem Soc 2006;128:11872-81.

[4] Tolosa J, Solntsev K, Tolbert L, Bunz U. Unsymmetrical cruciforms. J Org Chem 2010;75:523-34.

[5] Liu X, Sun Y, Hsu B, Lorbach A, Qi L, Heeger A, et al. Design and properties of intermediate-sized narrow band-gap conjugated molecules relevant to solution-processed organic solar cells. J Am Chem Soc 2014;136:5697-708.

[6] Wilson J, Bunz U. Switching of intramolecular charge transfer in cruciforms:metal 
ion sensing. J Am Chem Soc 2005;127:4124-5.

[7] Li Y, Wang D, Wang L, Li Z, Cui Q, Zhang H, et al. Novel asymmetrical pyrene derivatives as light emitting materials: synthesis and photophysics. J Lumin 2012;132:1010-4.

[8] Beverina L, Pagani G. $\pi$-Conjugated zwitterions as paradigm of donor-acceptor building blocks in organic-based materials. Acc Chem Res 2014;47:319-29.

[9] Li J, Liu T, Zheng M, Sun M, Zhang D, Zhang H, et al. Dibutylaminophenyland/or pyridinyl-capped 2,6,9,10-tetravinylanthracene cruciforms: synthesis and aggregation-enhanced one- and two-photon excited fluorescence. J Phys Chem C 2013;117:8404-10.

[10] Clare J, Tulevski G, Sugo K, Picciotto A, White K, Nuckolls C. Cruciform $\pi$-systems for molecular electronics applications. J Am Chem Soc 2003; 125: 6030-1. [11] Li H, Chi Z, Zhang X, Xu B, Liu S, Zhang Y, et al. New thermally stable aggregation-induced emission enhancement compounds for non-doped red organic light-emitting diodes. Chem Commun 2011;47:11273-5.

[12] Minaev B, Baryshnikovb G, Agrena H. Principles of phosphorescent organic light emitting devices. Phys Chem Chem Phys 2014;16:1719-58.

[13] Gil-Escrig L, Longo G, Pertegás A, Roldán-Carmona C, Soriano A, Sessolo, et al. Efficient photovoltaic and electroluminescent perovskite devices. Chem Commun 2015;51:569-71.

[14] Narayanan S, Abbas A, Raghunathan S, Sreekumar K, Karthab C, Joseph R. Theoretical and experimental investigations on the photoconductivity and nonlinear 
optical properties of donor-acceptor $\pi$-conjugated copolymer, poly $(2,5-(3,4-$ ethylenedioxythiophene)-alt-2,7-(9,9-dioctylfluorene)). RSC Adv 2015;5:8657-68.

[15] Wang H, Feng J, Wen G, Jiang H, Wan J, Zhu R, et al. Cruciform p-n diblock conjugated oligomers for electroluminescent applications. New J Chem 2006;30:667-70.

[16] Zhou X, Li H, Chi Z, Zhang X, Zhang J, Xu B, et al. Piezofluorochromism and morphology of a new aggregation-induced emission compound derived from tetraphenylethylene and carbazole. New J Chem 2012;36:685-93.

[17] Huang F, Tian Y, Chen C, Cheng Y, Young A, Jen A. Cross-conjugated polymers with large two-photon absorption cross-sections for metal ion sensing. J Phys Chem C 2007;111:10673-81.

[18] Zhang X, Chi Z, Xu B, Jiang L, Zhou X, Zhang Y, et al. Multifunctional organic fluorescent materials derived from 9,10-distyrylanthracene with alkoxyl endgroups of various lengths. Chem Commun 2012;48:10895-7.

[19] Zheng M, Sun M, Zhang D, Liu T, Xue S, Yang W. Fluorescence response of pyridinyl- and/or dibutylaniline-capped 2,6,9,10-tetravinylanthracenes to metal ions. Dyes Pigm 2014;101:101-9.

[20] Bilge A, Zen A, Forster M, Li H, Galbrecht F, Nehls B, et al. Swivel-cruciform oligothiophene dimers. J Mater Chem 2006;16:3177-82.

[21] Spitler E, Shirtcliff L, Halley M. Systematic structureeproperty investigations and ion-sensing studies of pyridine-derivatized donor/acceptor tetrakis (arylethynyl) benzenes. J Org Chem 2007;72:86-96. 
[22] Li X, Zhang X, Li W, Wang Y, Liu T, Zhang B, et al. Synthesis and enhanced two-photon absorption properties of tetradonor-containing anthracene-centered 2-D cross-conjugated polymers. J Mater Chem 2011;21:3916-24.

[23] Sun X, Liu Y, Chen S, Qiu W, Yu G, Ma Y, et al. X-shaped electroactive molecular materials based on oligothiophene architectures: facile synthesis and photophysical and electrochemical properties. Adv Funct Mater 2006;16:917-25.

[24] Xing L, Yang B, Wang X, Wang J, Chen B, Wu Q, et al. Probing the hydrophobic interactions of a series of pyrene end-labeled poly(ethyleneoxide)s in aqueous solution using time-resolved fluorescence. Langmuir 2013;29:2843-8.

[25] Liang J, Hu F, Lv X, Chen Z, Chen Z, Yin J. Synthesis, characterization and mechanochromic behavior of binuclear gold(I) complexes with various diisocyano bridges. Dyes Pigm 2012;95:485-90.

[26] Gong Y, Tan Y, Liu J, Lu P, Feng C, Yuan W, et al. Twisted D- $\pi$-A solid emitters: efficient emission and high contrast mechanochromism. Chem Commun 2013;49:4009-11.

[27] Mizuguchi K, Nakano H. Mechanofluorochromism of 4-[bis(9,9-dimethyl fluoren-2-yl)amino]benz-aldehyde. Dyes Pigm 2013;96:76-80.

[28] Zhang H, Guo E, Zhang Y, Ren P, Yang W. Donor-acceptor-substituted anthracene-centered cruciforms: synthesis, enhanced two-photon absorptions, and spatially separated frontier molecular orbitals. Chem Mater 2009;21:5125-35. [29] Crosby G, Demas J. Measurement of photoluminescence quantum yields. J Phys Chem 1971;75:991-1024. 
[30] Wang Z, Feng Y, Li H, Gao Z, Zhang X, Lu P, et al. Dimeric

411 phenanthroimidazole for blue electroluminescent materials: the effect of substituted

412 position attached to biphenyl center. Phys Chem Chem Phys 2014;16:10837-43.

413 [31] Wang Z, Song X, Gao Z, Yu D, Zhang X, Lu P et al. Tuning of the electronic and 414 optical properties of 4,4'-bis(1-phenylphenanthro[9,10-d]imidazol-2-yl)biphenyl via 415 cyano substitution in un-conjugated phenyl. RSC Advances 2012;2:9635-42.

[32] Wang M, Song X, Ma L, Feng Y, Gu C, Zhang X, et al. A triphenylamine-capped solution-processable wholly aromatic organic molecule with electrochemical stability and its potential application in photovoltaic devices. New J Chem 2013;37:2440-47.

Phenothiazine cruciforms: synthesis and metallochromic properties. J Org Chem 2007;72:6714-25.

[34] Jang J, Park H, Shin M, Kang H, Oha D, Jung S, et al. Novel quinoxaline derivatives containing arylaminated aceanthrylene for organic red-light emitting diodes. Dyes Pigm 2011;88:44-49. 
Scheme 1. The structure and synthetic route of anthracene-centered cruciform (FCA) studied in this paper.

Fig. 1 The absorption and emission spectra of FCA solution in THF at $1.0 \times 10^{-5} \mathrm{M}$.

Fig. 2 The emission spectra of FCA in THF/water mixtures at $1.0 \times 10^{-5} \mathrm{M}$.

Fig. 3 The absorption and emission spectra of FCA under pristine (as-prepared) and ground solid-states.

Fig. 4 The thermogravimetric (TGA) curve of FCA solid and the differential scanning calorimetric (DSC) curves of FCA solids under pristine (as-prepared) and ground solid-states (inset). $\mathrm{nm}) /$ FCA $(60 \mathrm{~nm}) / \mathrm{TPBi}(40 \mathrm{~nm}) / \mathrm{LiF}(0.5 \mathrm{~nm}) / \mathrm{Al}(100 \mathrm{~nm})$.

Fig. 5 The cyclic voltammetry (CV) curves of FCA in MeCN.

Fig. 6 Current density-luminance-voltage curves of the device ITO/PEDOT:PSS (40

Fig. 7 The luminous efficiency-power efficiency-voltage curves of the device ITO/PEDOT:PSS $(40 \mathrm{~nm}) /$ FCA $(60 \mathrm{~nm}) / \mathrm{TPBi}(40 \mathrm{~nm}) / \mathrm{LiF}(0.5 \mathrm{~nm}) / \mathrm{Al}(100 \mathrm{~nm})$.

The inset is the electro- luminescent spectrum. 


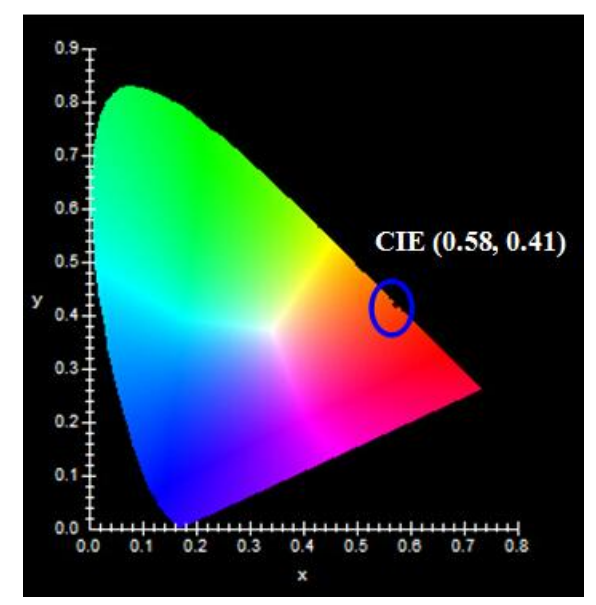

Figure R1. The CIE of device at $6 \mathrm{~V}$.

Comment 3: The state of the current non-doped OLEDs based on red fluorescent dyes should be discussed in more detail and compared with your results.

Response: We agree with the reviewer's comments. We have supplied the relative references in ref. 34 to compare with our results.

Comment 4: The film quality is very important to the performance of the materials in EL devices. If technologically possible, it is better to measure the film quality. This may be helpful to understand why the EL efficiency is not so high.

Response: We agree with the reviewer's comments. We have measured the atomic force microscopy (AFM) image of the emitting material atop ITO-glass to evaluate the film quality (see the following Figure R2). The emitting material film is pinhole-free and quite smooth with a surface roughness (root-mean-square) of approximately $0.481 \mathrm{~nm}$, and it showed that the film quality of the emitting material meet the basic requirements for solution-processed fluorescent small-molecular OLEDs. 


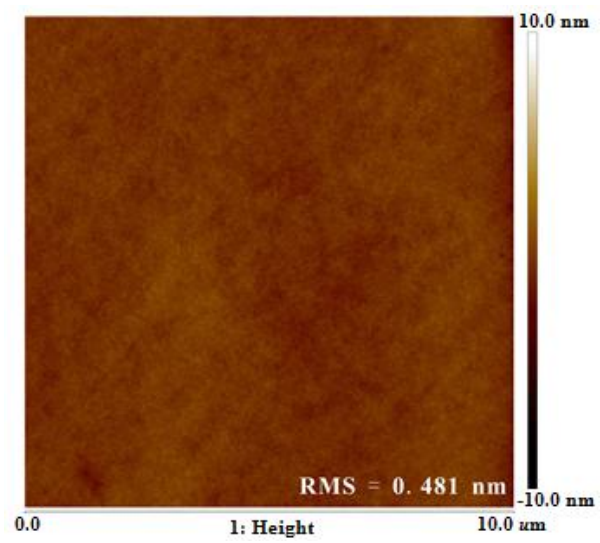

Figure R2. $10 \mu \mathrm{m} \times 10 \mu \mathrm{m}$ AFM image of spin-coated film (the emitting material deposited atop ITO-glass).

Thank you very much. Should you have any questions, please contact us without hesitate.

Sincerely yours,

Professor Wenjun Yang

Key Laboratory of Rubber-plastics (QUST), Ministry of Education,

School of Polymer Science and Engineering,

Qingdao University of Science \& Technology,

53-Zhengzhou Road, Qingdao, 266042, P. R. China.

Fax: 86-532-84023977, Email: ywjph2004@qust.edu.cn 


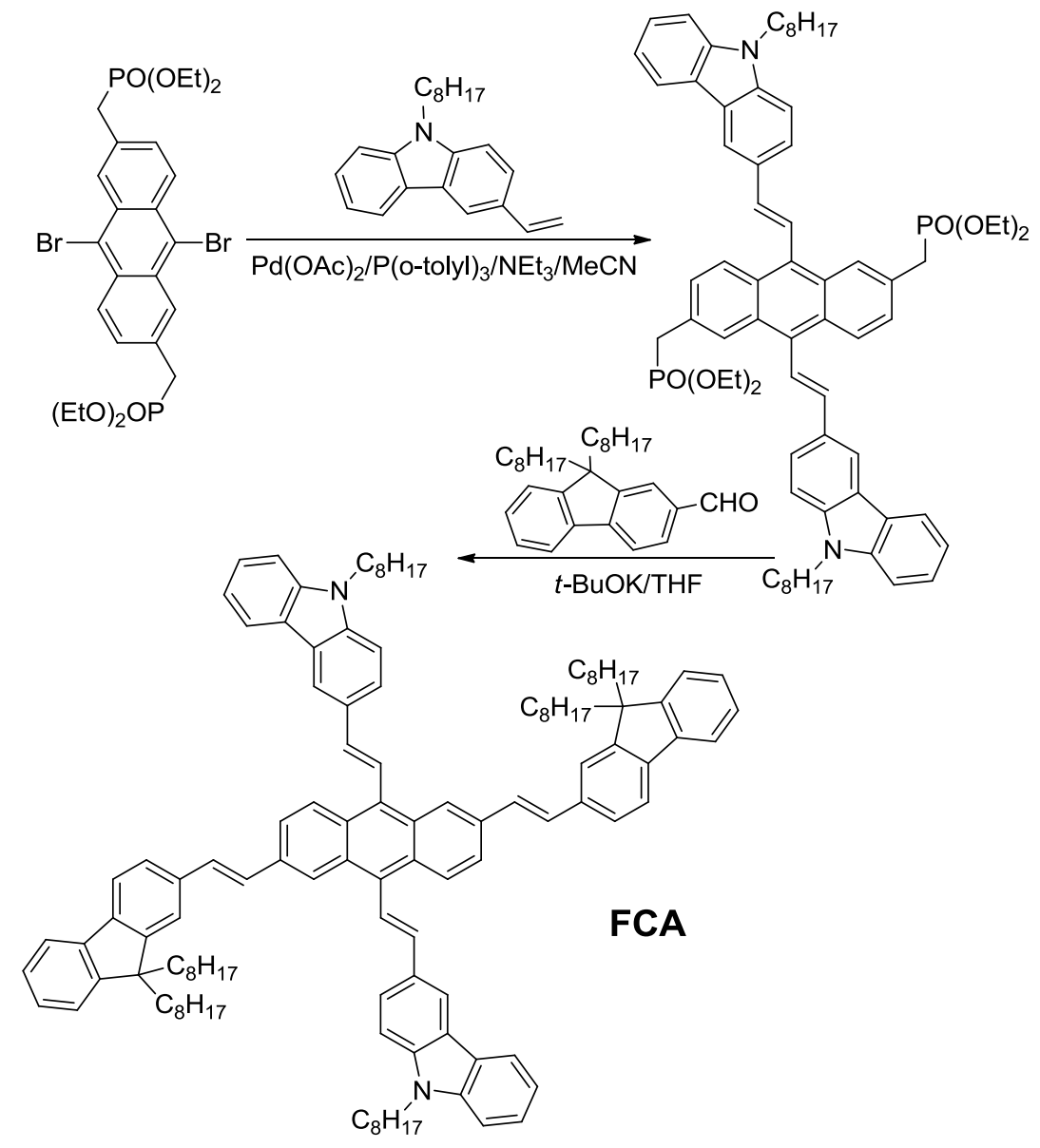

Scheme 1. The structure and synthetic route of anthracene-centered cruciform (FCA) studied in this paper. 


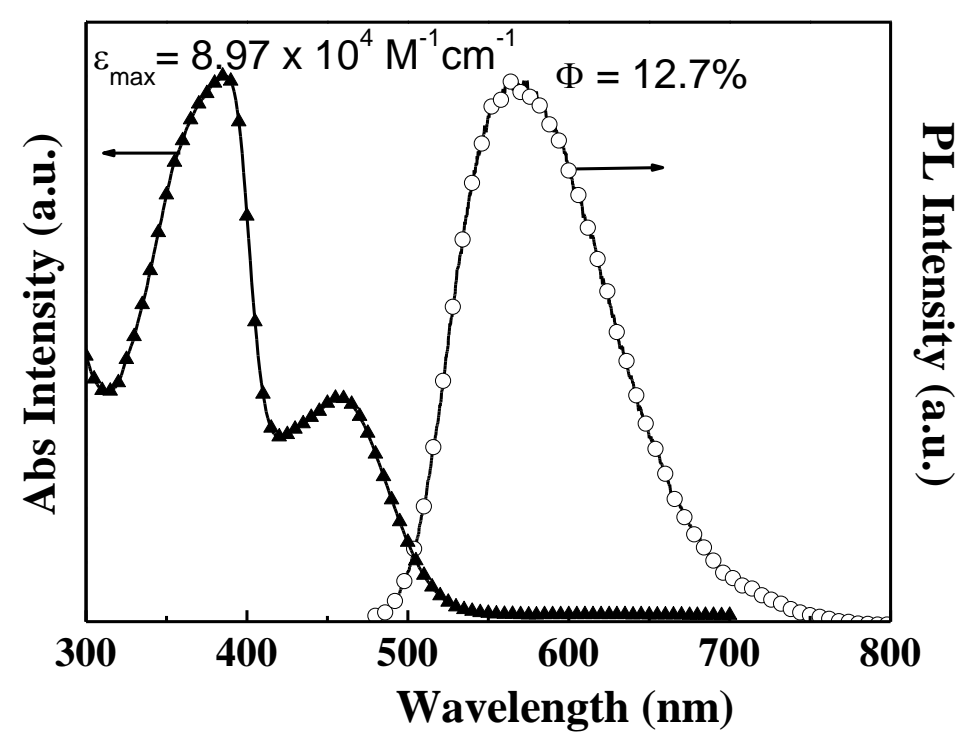

Fig. 1 The absorption and emission spectra of FCA solution in THF at $1.0 \times 10^{-5} \mathrm{M}$. 


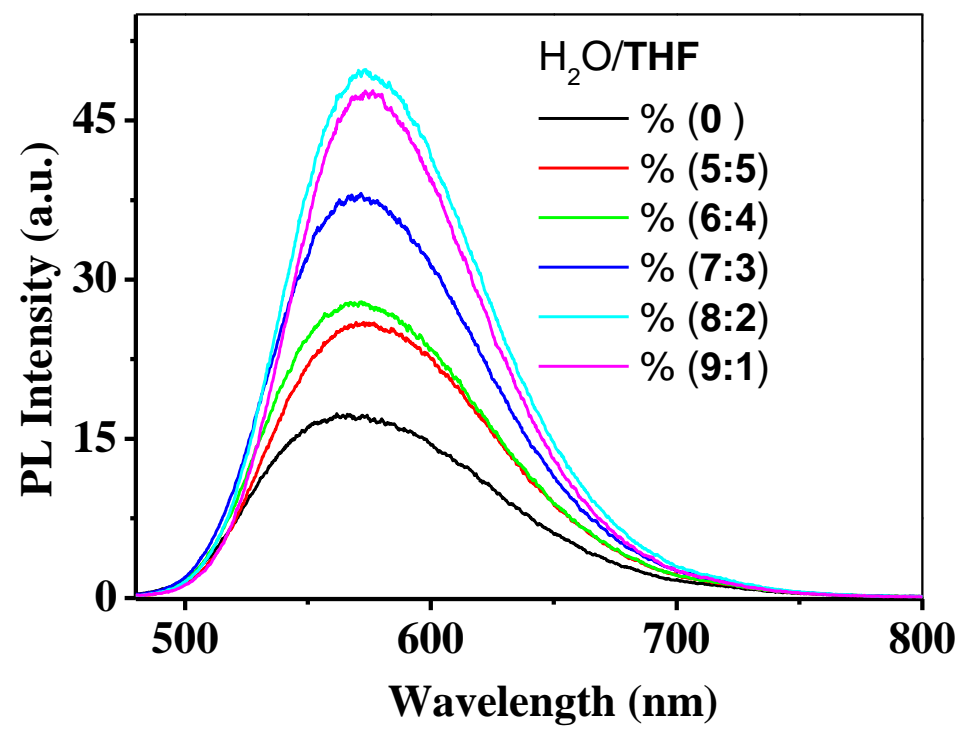

Fig. 2 The emission spectra of FCA in THF/water mixtures at $1.0 \times 10^{-5} \mathrm{M}$. 


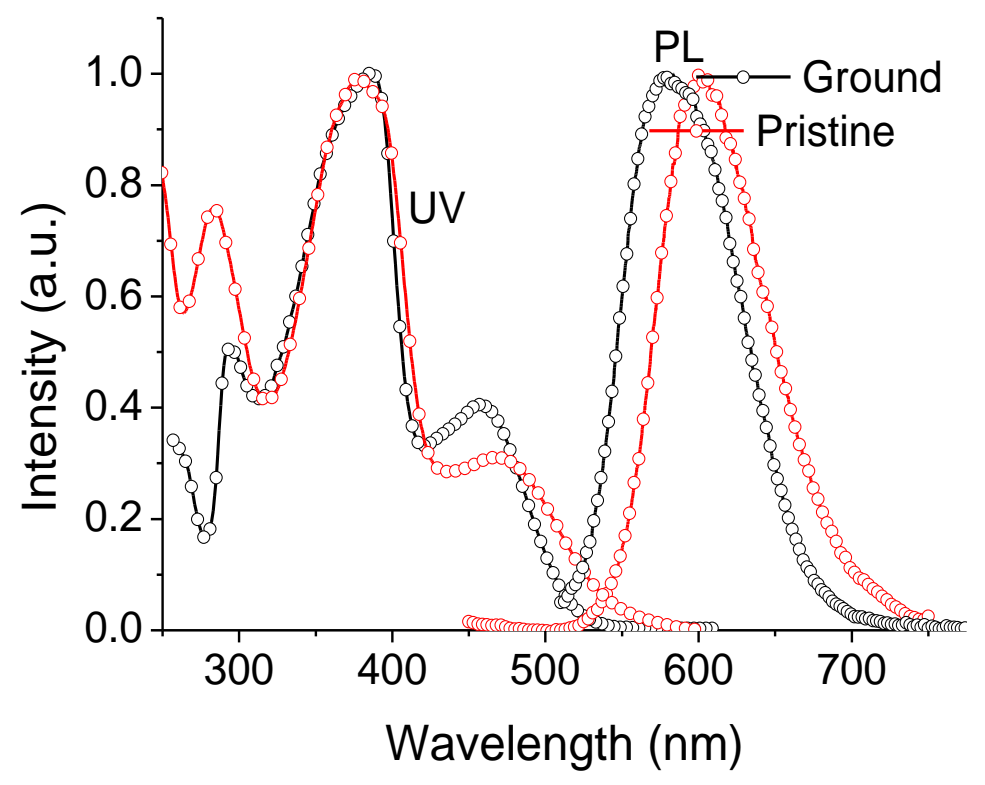

Fig. 3 The absorption and emission spectra of FCA under pristine (as-prepared) and ground solid-states. 


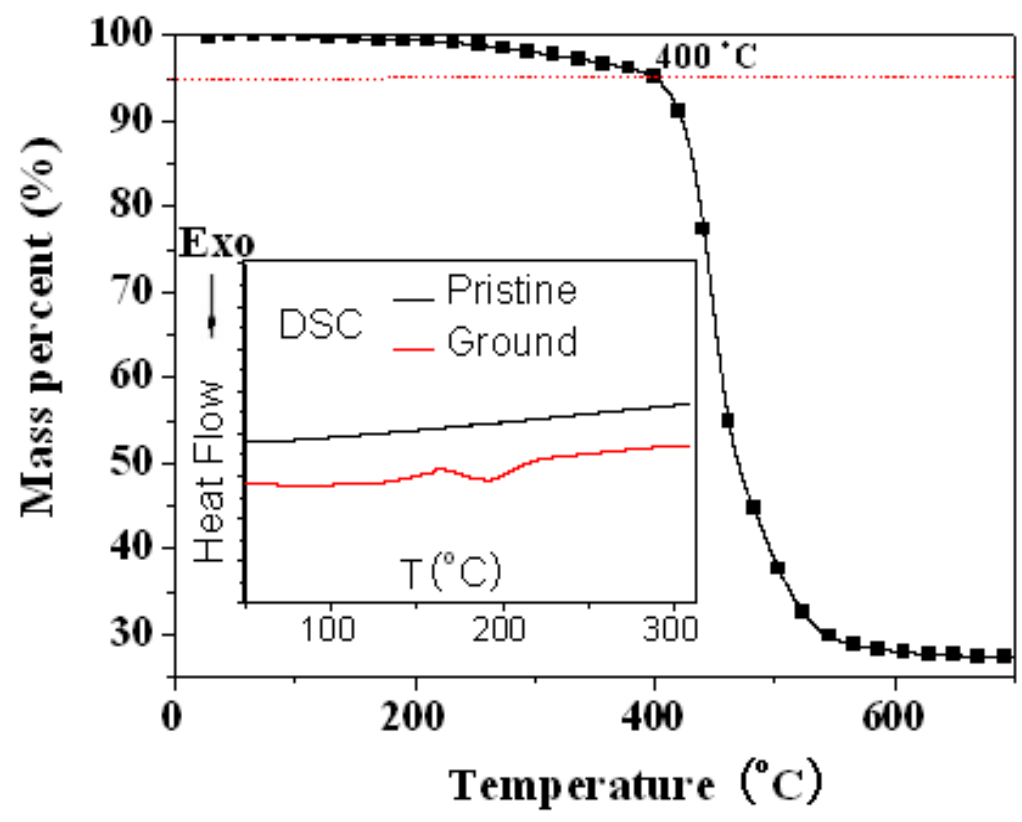

Fig. 4 The thermogravimetric (TGA) curve of FCA solid and the differential scanning calorimetric (DSC) curves of FCA solids under pristine (as-prepared) and ground solid-states (inset). 


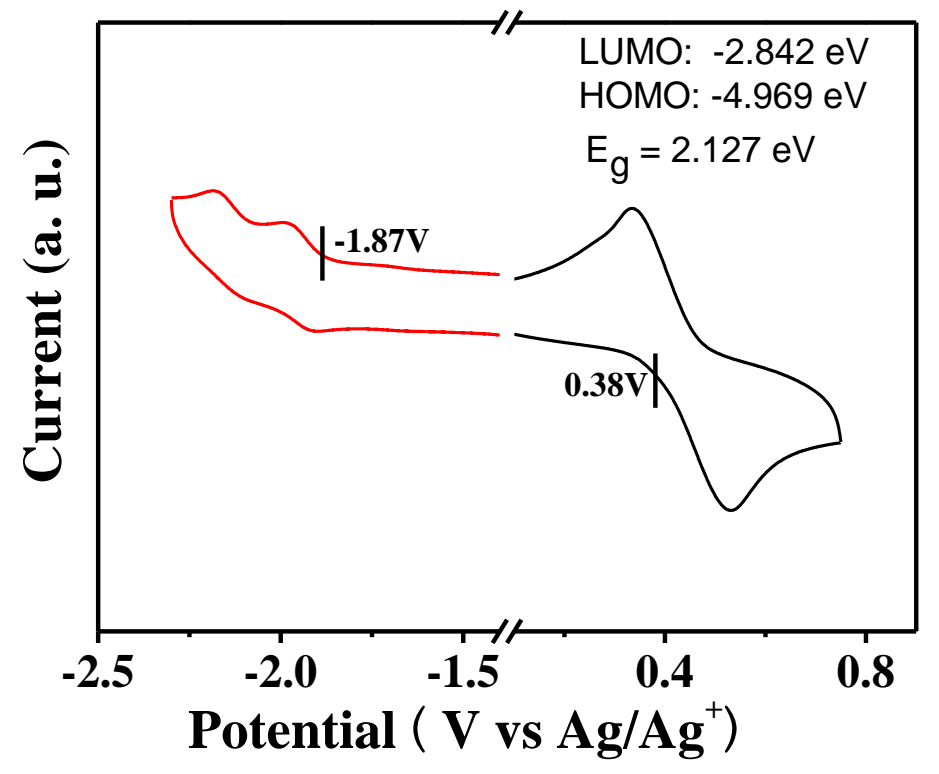

Fig. 5 The cyclic voltammetry (CV) curves of FCA in $\mathrm{MeCN}$ 


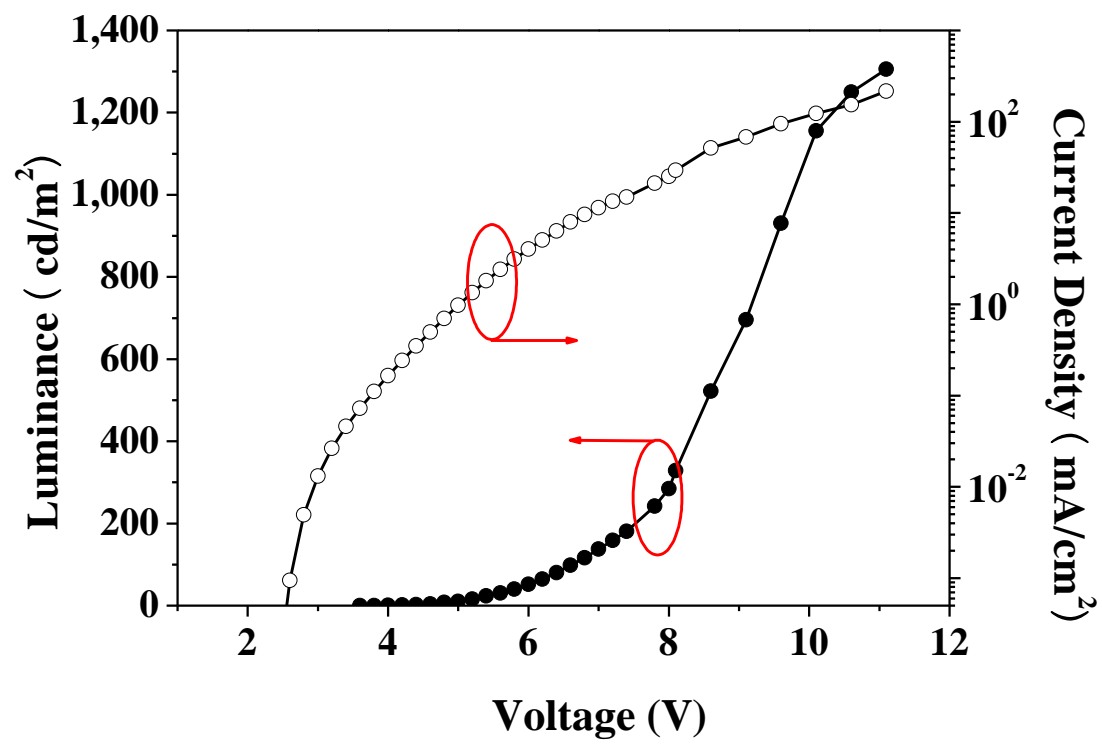

Fig. 6 Current density-luminance-voltage curves of the device ITO/PEDOT:PSS (40 $\mathrm{nm}) /$ FCA $(60 \mathrm{~nm}) / \mathrm{TPBi}(40 \mathrm{~nm}) / \mathrm{LiF}(0.5 \mathrm{~nm}) / \mathrm{Al}(100 \mathrm{~nm})$. 


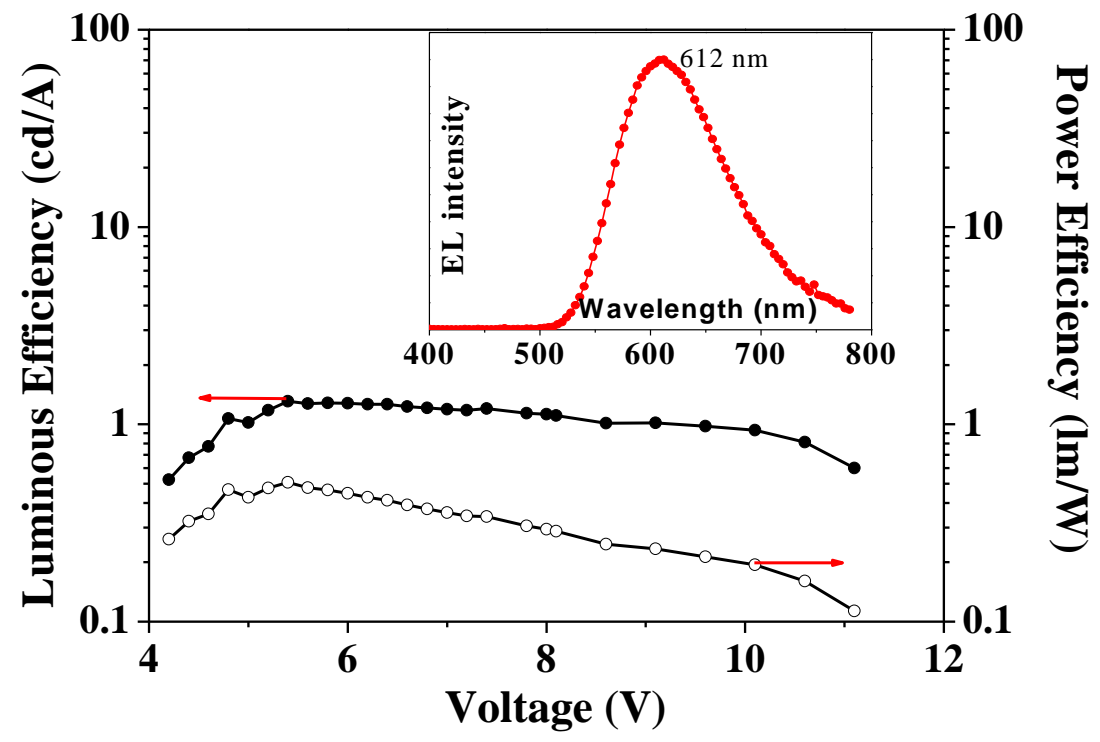

Fig. 7 The luminous efficiency-power efficiency-voltage curves of the device ITO/PEDOT:PSS $(40 \mathrm{~nm}) /$ FCA $(60 \mathrm{~nm}) / \mathrm{TPBi}(40 \mathrm{~nm}) / \mathrm{LiF}(0.5 \mathrm{~nm}) / \mathrm{Al}(100 \mathrm{~nm})$. The inset is the electro- luminescent spectrum. 